FISIST/2-98/CFIF

\title{
Quasi degenerate Neutrino Masses with Universal Strength Yukawa Couplings
}

\author{
G. C. Branco, M. N. Rebelo ${ }^{\dagger}$ and J. I. Silva-Marcos ${ }^{\ddagger}$ \\ CFIF/IST and Departamento de Física \\ Instituto Superior Técnico \\ Av. Rovisco Pais, \\ P-1096 Lisboa Codex, Portugal
}

\begin{abstract}
A simple ansatz is proposed for neutrino and charged lepton mass matrices, within the framework of universal strength for Yukawa couplings. In this framework all Yukawa couplings have equal moduli and the flavour dependence is only in their phases. We take into account the solar neutrino deficit and the atmospheric neutrino anomaly, assuming three neutrino families only. The ansatz leads in a natural way to small mixing involving neutrinos of quasi degenerate masses, as required to explain the solar neutrino deficit in the nonadiabatic MSW solution, while having the large mixing necessary to explain the atmospheric neutrino anomaly.
\end{abstract}

\section{Introduction}

Presently the Standard Model (SM) enjoys quite a remarkable success when confronted with experiment. However, there is recent experimental evidence pointing towards physics beyond the SM in the leptonic sector, to wit, the solar neutrino deficit, the atmospheric neutrino problem, and the results of the LSND collaboration suggesting that neutrino oscillations might have been observed in an accelerator experiment.

\footnotetext{
${ }^{*}$ E-mail address : d2003@beta.ist.utl.pt

${ }^{\dagger}$ E-mail address : rebelo@beta.ist.utl.pt

${ }_{\ddagger}^{\ddagger}$ E-mail address : juca@cfif1.ist.utl.pt
} 
The solar neutrino data obtained by several different experiments [1], indicate a deficit in the number of observed neutrinos by comparison to the Standard Solar Model (SSM) predictions for the solar neutrino fluxes [2]. The solar neutrino deficit is explained in terms of oscillations of the electron neutrino into some other neutrino species. In the framework of the MSW mechanism [3] there are two sets of solutions, the adiabatic branch (AMSW) requiring a large mixing $\left(\sin ^{2} 2 \theta \simeq 0.65 \sim 0.85\right.$ ) 田 and the non adiabatic branch (NAMSW) requiring a small mixing $\left(\sin ^{2} 2 \theta \simeq(0.1 \sim\right.$ 2) $\left.\times 10^{-2}\right)$ [4 with $\Delta m^{2} \simeq(0.3 \sim 1.2) \times 10^{-5} \mathrm{eV}^{2}$. The small mixing solution seems to be favoured by the present data. In the framework of vacuum oscillation only three separate regions within a narrow range of parameters $\left(\Delta \mathrm{m}^{2}=5-8 \times 10^{-11} \mathrm{eV}^{2}\right.$ and $\sin ^{2} 2 \theta=0.65-1$ ) are allowed [4].

Several experiments have measured the ratio of the number of muon neutrinos by the number of electron neutrinos produced in the atmosphere through the decay of pions and kaons with subsequent decay of secondary muons [5]. The combined results lead to,

$$
R \equiv \frac{\left(n_{\nu_{\mu}} / n_{\nu_{e}}\right)_{\mathrm{Data}}}{\left(n_{\nu_{\mu}} / n_{\nu_{e}}\right)_{\mathrm{SM}}} \simeq 0.6
$$

where $\left(n_{\nu_{\mu}} / n_{\nu_{e}}\right)_{\text {Data }}$ is the measured ratio of muon-neutrino to electron-neutrino events, while $\left(n_{\nu_{\mu}} / n_{\nu_{e}}\right)_{\mathrm{SM}}$ is the expected ratio assuming no oscillations. This anomaly can be caused by oscillations of the atmospheric muon neutrinos into another type of neutrino with large mixing angle $\left(\sin ^{2} 2 \theta \simeq 0.6 \sim 1.0, \Delta m^{2} \simeq(0.3 \sim 3) \times 10^{-2} \mathrm{eV}^{2}\right)$.

The LSND group has reported evidence for $\overline{\nu_{\mu}}-\overline{\nu_{e}}$ and $\nu_{\mu}-\nu_{e}$ oscillations [6]. However no other accelerator experiment confirmed their result thus strongly reducing the allowed parameter space. The resulting experimental constraints on neutrino masses and mixings are such that no combined solution to the solar, atmospheric and LSND results can be found in the framework of three neutrinos only, since the required neutrino mass differences do not satisfy the relation $\Delta m_{21}^{2}+\Delta m_{32}^{2}=\Delta m_{31}^{2}$. We have chosen to consider simply three neutrino families without additional sterile neutrinos and not to take into consideration the LSND data.

Astrophysical considerations, in particular the possibility that neutrinos constitute the hot dark matter, favour neutrino masses of the order of a few $e V$ [7], which combined with the above constraints leads to a set of highly degenerate neutrinos.

In this paper we propose a simple ansatz within the framework of universal strength for Yukawa couplings (USY) [8] which leads to quasi-degeneracy of neutrino masses and provides a solution to the solar and atmospheric neutrino problems. In USY all Yukawa couplings have equal moduli so that the flavour dependence is only contained in their phases. For the quark sector it has been shown [9], [10] that the USY hypothesis leads to a highly predictive and successful ansatz for quark masses and mixings. Various mixing schemes for the leptonic sector have been suggested [11]. The possibility of quasi-degenerate neutrino masses has been recently considered in the literature in the context of some specific symmetry or ansatz. An important feature of our ansatz lies on the fact that it can accommodate in a natural way small mixing involving a set of highly degenerate neutrinos in the MSW solution 
to the solar neutrino problem while also having the large mixing necessary to explain the atmospheric neutrino data.

\section{Degeneracy in USY}

The USY hypothesis leads to neutrino mass matrices of the form:

$$
M_{\nu}=c_{\nu}\left[e^{i \theta_{i j}}\right]
$$

where $c_{\nu}$ is an overall constant. Let us derive the conditions which should be satisfied so that the matrices of Eq.(2) lead to at least two degenerate neutrinos. It is useful to introduce the dimensionless Hermitian matrix $H_{\nu} \equiv M_{\nu} M_{\nu}^{\dagger} / 3 c_{\nu}^{2}$ which can be written as:

$$
H_{\nu}=\left[\begin{array}{lll}
1 & r_{1} e^{i \varphi_{1}} & r_{2} e^{i \varphi_{2}} \\
r_{1} e^{-i \varphi_{1}} & 1 & r_{3} e^{i \varphi_{3}} \\
r_{2} e^{-i \varphi_{2}} & r_{3} e^{-i \varphi_{3}} & 1
\end{array}\right]
$$

where the off diagonal elements $r_{i} e^{i \varphi_{i}}$ are the sum of products of pure phase elements of $M_{\nu}$ :

$$
\left(H_{\nu}\right)_{12}=r_{1} e^{i \varphi_{1}}=\frac{1}{3}\left[e^{i\left(\theta_{11}-\theta_{21}\right)}+e^{i\left(\theta_{12}-\theta_{22}\right)}+e^{i\left(\theta_{13}-\theta_{23}\right)}\right]
$$

with analogous expressions for $\left(H_{\nu}\right)_{13}$ and $\left(H_{\nu}\right)_{23}$. It can be readily verified that if at least two of the eigenvalues of $H_{\nu}$ are equal, then the following relation holds:

$$
\left[1-\frac{\chi}{3}\right]^{3}=\left[1-\frac{\chi-\delta}{2}\right]^{2}
$$

where:

$$
\begin{aligned}
& \delta \equiv \operatorname{det}\left(H_{\nu}\right)=\lambda_{1} \lambda_{2} \lambda_{3} \\
& \chi \equiv \chi\left(H_{\nu}\right)=\lambda_{1} \lambda_{2}+\lambda_{1} \lambda_{3}+\lambda_{2} \lambda_{3}
\end{aligned}
$$

and the $\lambda_{i}=3 m_{i}^{2} /\left(m_{1}^{2}+m_{2}^{2}+m_{3}^{2}\right)$ denote the eigenvalues of $H_{\nu}$. In the derivation of Eq.(5) we took into account that $\operatorname{tr}\left(H_{\nu}\right) \equiv \lambda_{1}+\lambda_{2}+\lambda_{3}=3$. The invariants $\chi$ and $\delta$ can be expressed in terms of $r_{i}$ and $\varphi_{i}$, with all generality, as:

$$
\begin{aligned}
& \chi=3-r_{1}^{2}-r_{2}^{2}-r_{3}^{2} \\
& \delta=1+2 r_{1} r_{2} r_{3} \cos \left(\varphi_{1}+\varphi_{3}-\varphi_{2}\right)-r_{1}^{2}-r_{2}^{2}-r_{3}^{2}
\end{aligned}
$$

The following "spherical" parametrization for the $r_{i}$ is useful:

$$
\begin{array}{ll}
r_{1}=[3(1-\chi / 3)]^{1 / 2} & \sin \theta \cos \phi \\
r_{2}=[3(1-\chi / 3)]^{1 / 2} & \sin \theta \sin \phi \\
r_{3}=[3(1-\chi / 3)]^{1 / 2} & \cos \theta
\end{array}
$$

with $0 \leq \theta, \phi \leq \frac{\pi}{2}$. From Eqs.(7) and (8), one obtains: 


$$
\sin ^{2} \theta \cos \theta \cdot \sin (2 \phi) \cdot \cos \left(\varphi_{1}+\varphi_{3}-\varphi_{2}\right)=\frac{2}{3 \sqrt{3}}\left[1-\frac{\chi-\delta}{2}\right] /\left[1-\frac{\chi}{3}\right]^{\frac{3}{2}}
$$

In the case of at least two degenerate neutrinos Eq.(9) can be combined with Eq.(5) leading to the condition:

$$
\sin ^{2} \theta \cos \theta \cdot \sin (2 \phi) \cdot \cos \left(\varphi_{1}+\varphi_{3}-\varphi_{2}\right)=\frac{2}{3 \sqrt{3}}
$$

which can only be satisfied for $\cos \theta=1 / \sqrt{3}, \sin (2 \phi)=1$ and $\cos \left(\varphi_{1}+\varphi_{3}-\varphi_{2}\right)=1$. Therefore, the necessary and sufficient conditions for $H_{\nu}$ to have two degenerate eigenvalues are:

$$
\begin{aligned}
& \varphi_{1}+\varphi_{3}-\varphi_{2}=0(\bmod .2 \pi) \\
& r_{1}=r_{2}=r_{3}
\end{aligned}
$$

Within the USY framework, it can be shown that for matrices $M_{\nu}$ having at least two degenerate eigenvalues, there is a weak-basis where $M_{\nu}$ has one of the following forms (modulo trivial permutations)

$$
\begin{aligned}
& M_{\nu}^{\mathrm{I}}=c_{\nu} K \cdot\left[\begin{array}{lll}
e^{i \alpha} & 1 & 1 \\
1 & e^{i \alpha} & 1 \\
1 & 1 & e^{i \alpha}
\end{array}\right] ; M_{\nu}^{\mathrm{II}}=c_{\nu} K \cdot\left[\begin{array}{lll}
e^{i \alpha} & 1 & 1 \\
1 & e^{i \alpha} & 1 \\
1 & 1 & e^{-2 i \alpha}
\end{array}\right] \\
& M_{\nu}^{\mathrm{III}}=c_{\nu} K \cdot\left[\begin{array}{lll}
e^{i \alpha} & 1 & 1 \\
1 & -1 & 1 \\
1 & 1 & -1
\end{array}\right]
\end{aligned}
$$

where $K \equiv \operatorname{diag}\left(e^{i \varphi_{1}}, e^{i \varphi_{2}}, e^{i \varphi_{3}}\right)$ and $c_{\nu}$ is a real constant.

The first two cases of Eq.(12) are of special interest since for $\alpha=2 \pi / 3$ they lead to three degenerate neutrino masses, while for $\alpha \neq 2 \pi / 3$ they lead to only two degenerate mass eigenvalues.

\section{A Special Ansatz}

In order to have an ansatz with predictions for the leptonic mixing matrix, one has to specify the structure of the charged lepton mass matrix $M_{\ell}$ together with the structure of $M_{\nu}$.

Our guiding principle is the assumption that all leptonic Yukawa couplings obey the USY hypothesis. Furthermore, we choose, within USY, the same structure of phases for the charged lepton and neutrino mass matrices. Guided by the above ideas we propose the following specific ansatz:

$$
M_{\ell}=c_{\ell}\left[\begin{array}{lll}
e^{-i a} & 1 & 1 \\
1 & e^{i a} & 1 \\
1 & 1 & e^{i b}
\end{array}\right] \quad ; M_{\nu}=c_{\nu}\left[\begin{array}{lll}
e^{i \alpha} & 1 & 1 \\
1 & e^{i \alpha} & 1 \\
1 & 1 & e^{i \beta}
\end{array}\right]
$$


with $c_{\ell}, c_{\nu}$ real constants.

The leptonic mixing matrix $V$ appearing in the charged weak current is then given by

$$
V=U_{\nu}^{\dagger} \cdot U_{\ell}
$$

where the matrix $U_{\ell}$ diagonalizes $M_{\ell} M_{\ell}^{\dagger}$ and the matrix $U_{\nu}$ diagonalizes $M_{\nu} M_{\nu}^{\dagger}$ in the case of Dirac neutrinos . Note that both $M_{\ell}$ and $M_{\nu}$ have only three parameters each. As a result, both $U_{\ell}$ and $U_{\nu}$ will be entirely fixed by charged lepton and neutrino mass ratios. Due to the observed strong hierarchy in the charged lepton mass spectrum, the parameters $(a, b)$ will be close to zero. From the form of $M_{\ell}$ in Eq.(13) one can derive exact expressions for the phases $(a, b)$ in terms of charged lepton mass ratios. In leading order, one obtains:

$$
a=3 \sqrt{3} \frac{\sqrt{m_{e} m_{\mu}}}{m_{\tau}} \quad ; \quad b=\frac{9}{2} \frac{m_{\mu}}{m_{\tau}}
$$

In the neutrino sector, it was already pointed out that the square mass differences necessary to explain the solar neutrino data, together with the requirement that neutrinos constitute the hot dark matter, lead to highly degenerate neutrinos. The matrix $M_{\nu}$ in Eq.(13) leads to threefold degeneracy for $\alpha=\beta=2 \pi / 3$. Thus, it is useful to introduce the two small parameters $\epsilon_{32}, \epsilon_{21}$, defined by:

$$
\alpha=\frac{2 \pi}{3}-\epsilon_{32}-\epsilon_{21} \quad ; \quad \beta=\frac{2 \pi}{3}+2 \epsilon_{32}
$$

It is clear from this parametrization, that in the limit $\epsilon_{21}=0$, one has for the phase $\beta=-2 \alpha(\bmod 2 \pi)$, and therefore one obtains in this limit the mass matrix $M_{\nu}^{\mathbb{I I}}$ of Eq.(12), with two exactly degenerate masses. Using Eq.(13), one calculates the eigenvalues $\lambda_{i}$ of $H_{\nu}=M_{\nu} M_{\nu}^{\dagger} / 3 c_{\nu}^{2}$ as functions of $\alpha$ and $\beta$,

$$
\lambda_{1}=1-y ; \quad \lambda_{2}=\frac{2+y-\sqrt{8 x^{2}+y^{2}}}{2} ; \quad \lambda_{3}=\frac{2+y+\sqrt{8 x^{2}+y^{2}}}{2}
$$

where $y=(2 \cos (\alpha)+1) / 3$ and $x=(1 / 3)[3+2 \cos (\alpha)+2 \cos (\beta)+2 \cos (\alpha+\beta)]^{1 / 2}$. Then from Eq.(17) one can express $\epsilon_{32}, \epsilon_{21}$ in terms of mass ratios. In leading order, one obtains:

$$
\epsilon_{32}=\frac{1}{\sqrt{3}} \frac{\Delta m_{32}^{2}}{m_{3}^{2}} \quad ; \quad \epsilon_{21}=\frac{3 \sqrt{3}}{4} \frac{\Delta m_{21}^{2}}{m_{3}^{2}}
$$

In order to evaluate the leptonic mixing matrix $V$, it is convenient to start by making a weak-basis (WB) transformation defined by:

\footnotetext{
${ }^{1}$ If both Dirac mass terms $\left(M_{D}\right)$ and Majorana mass terms for the righthanded neutrinos $\left(M_{R}\right)$ are present, with the light Majorana neutrinos acquiring mass through the seesaw mechanism [12], we identify $M_{\nu}$ of Eq.(12) with the effective mass matrix for the light neutrinos given by $M_{\nu}=M_{D} M_{R}^{-1} M_{D}^{T}$ and, in this case, $U_{\nu}$ is very approximately the matrix verifying $M_{\nu}=U_{\nu} \cdot \operatorname{diag}\left(m_{\nu_{1}}, m_{\nu_{2}}, m_{\nu_{3}}\right) \cdot U_{\nu}^{T}$
} 


$$
\begin{aligned}
& H_{\ell} \equiv \frac{1}{3 c_{\ell}^{2}} M_{\ell} M_{\ell}^{\dagger} \quad \longrightarrow \quad H_{\ell}^{\prime}=F^{\dagger} \cdot H_{\ell} \cdot F \\
& H_{\nu} \equiv \frac{1}{3 c_{\nu}{ }^{2}} M_{\nu} M_{\nu}^{\dagger} \longrightarrow H_{\nu}^{\prime}=F^{\dagger} \cdot H_{\nu} \cdot F
\end{aligned}
$$

where

$$
F=\left[\begin{array}{lll}
\frac{1}{\sqrt{2}} & \frac{-1}{\sqrt{6}} & \frac{1}{\sqrt{3}} \\
\frac{-1}{\sqrt{2}} & \frac{-1}{\sqrt{6}} & \frac{1}{\sqrt{3}} \\
0 & \frac{2}{\sqrt{6}} & \frac{1}{\sqrt{3}}
\end{array}\right]
$$

Obviously, after this WB transformation the charged leptonic weak current remains diagonal, real, i.e., $V=\mathbb{1}$. This WB transformation corresponds to writing $H_{\ell}$ in a "heavy basis". Due to the strong hierarchy of the charged lepton masses, in this "heavy basis" all other elements of $H_{\ell}^{\prime}$ are small, compared to the element $(3,3)$. The Hermitian matrix $H_{\ell}^{\prime}$ can then be diagonalized, and one obtains in leading order:

$$
\begin{array}{lll}
\left|U_{12}^{\ell}\right|=\sqrt{\frac{m_{e}}{m_{\mu}}} & ; & \left|U_{13}^{\ell}\right|=\frac{\sqrt{2 m_{e} m_{\mu}}}{m_{\tau}} \\
\left|U_{31}^{\ell}\right|=\frac{3}{\sqrt{2}} \frac{\sqrt{m_{e} m_{\mu}}}{m_{\tau}} & ; & \left|U_{23}^{\ell}\right|=\frac{1}{\sqrt{2}} \frac{m_{\mu}}{m_{\tau}}
\end{array}
$$

In the neutrino sector, one has a drastically different situation. After the WB transformation of Eq.(19), the first neutrino exactly decouples from the other two, i.e., $\left(H_{\nu}^{\prime}\right)_{12}=\left(H_{\nu}^{\prime}\right)_{13}=0$.

Furthermore, the mixing between the second and third neutrino state is large and, to leading order, independent of neutrino mass ratios, one obtains:

$$
U_{\nu}=\left[\begin{array}{ccc}
1 & 0 & 0 \\
0 & \frac{\omega^{\star}-\omega}{3} & \frac{\sqrt{2}\left(\omega^{\star}-1\right)}{3} \\
0 & \frac{\sqrt{2}\left(\omega^{\star}-1\right)}{3} & \frac{1-\omega}{3}
\end{array}\right]
$$

where $\omega=e^{i 2 \pi / 3}$. In Eq.(22) the zeros are exact yet we have omitted in the other entries terms of the order $\Delta m_{21}^{2} / \Delta m_{32}^{2}$. Therefore, the leptonic mixing matrix is given, in leading order, by:

$$
|V|=\left[\begin{array}{ccc}
1 & \sqrt{\frac{m_{e}}{m_{\mu}}} & \frac{\sqrt{2 m_{e} m_{\mu}}}{m_{\tau}} \\
\sqrt{\frac{m_{e}}{3 m_{\mu}}} & \frac{1}{\sqrt{3}} & \frac{\sqrt{2}}{\sqrt{3}} \\
\sqrt{\frac{2 m_{e}}{3 m_{\mu}}} & \frac{\sqrt{2}}{\sqrt{3}} & \frac{1}{\sqrt{3}}
\end{array}\right]
$$

\section{Confronting the data}

Neutrino oscillations [13 have to be taken into account since neutrino experiments are discussed in terms of neutrino weak eigenstates rather than physical states. With 
the notation $\nu_{\alpha(\beta)}$ for weak eigenstates and $\nu_{i(j)}$ for mass eigenstates, the probability of finding $\nu_{\beta}$ at time $t$ having started with $\nu_{\alpha}$ at $t=0$ neglecting the effect of $\mathrm{CP}$ violation in the leptonic sector (real $V$ ) is given by:

$$
\begin{gathered}
P\left(\nu_{\alpha} \rightarrow \nu_{\beta}\right) \equiv<\nu_{\beta}\left|\nu_{\alpha}(t)>\cdot<\nu_{\beta}\right| \nu_{\alpha}(t)>^{\star}= \\
\quad=\delta_{\alpha \beta}-4 \sum_{i<j} V_{\alpha i} V_{\beta i} V_{\alpha j} V_{\beta j} \cdot \sin ^{2}\left[\frac{\Delta m_{j i}^{2}}{4} \frac{L}{E}\right]
\end{gathered}
$$

where $E$ is the neutrino energy, $L$ is the distance travelled by the neutrino between the source and the detector, $V$ is the leptonic mixing matrix given by Eq.(14), and $\Delta m_{j i}^{2}$ is defined by:

$$
\Delta m_{j i}^{2}=\left|m_{j}^{2}-m_{i}^{2}\right|
$$

The interpretation of the experimental data is presented in terms of two flavour mixing, in this case Eq.(24) reduces to

$$
P\left(\nu_{\alpha} \rightarrow \nu_{\beta}\right)=\delta_{\alpha \beta}-\sin ^{2} 2 \theta \cdot \sin ^{2}\left[\frac{\Delta m_{j i}^{2}}{4} \frac{L}{E}\right]
$$

hence the meaning of the experimental bounds presented in section 1 .

The translation of the bounds into the three flavour approach is quite simple for the experimental limits imposed on $\Delta m_{j i}^{2}$, with $\Delta m_{21}^{2}$ in the solar range and $\Delta m_{32}^{2}$ in the atmospheric range. In the case of the atmospheric anomaly it is clear that we can disregard the term in $\sin ^{2}\left[\left(\Delta m_{21}^{2} / 4\right)(L / E)\right]$ and we can approximately write

$$
\sin ^{2}\left[\frac{\Delta m_{31}^{2}}{4} \frac{L}{E}\right] \simeq \sin ^{2}\left[\frac{\Delta m_{32}^{2}}{4} \frac{L}{E}\right] \equiv S
$$

so that

$$
1-P\left(\nu_{\mu} \rightarrow \nu_{\mu}\right)=P\left(\nu_{\mu} \rightarrow \nu_{e}\right)+P\left(\nu_{\mu} \rightarrow \nu_{\tau}\right)=4\left(V_{23} V_{23} V_{21} V_{21}+V_{22} V_{22} V_{23} V_{23}\right) S
$$

and we identify

$$
\sin ^{2} 2 \theta_{\mathrm{atm}}=4\left(V_{23} V_{23} V_{21} V_{21}+V_{22} V_{22} V_{23} V_{23}\right)
$$

In the case of the solar neutrino anomaly the range $L / E$ is such that $S$ in Eq.(27) can be averaged to $\frac{1}{2}$ and we obtain

$$
1-P\left(\nu_{e} \rightarrow \nu_{e}\right)=4\left(V_{11} V_{11} U_{13} U_{13}+V_{12} V_{12} V_{13} V_{13}\right) \cdot \frac{1}{2}+4 V_{11} V_{11} V_{12} V_{12} \sin ^{2}\left[\frac{\Delta m_{32}^{2}}{4} \frac{L}{E}\right]
$$

in our numerical example the first term of this equation is small so, as a result, we can identify

$$
\sin ^{2} 2 \theta_{\text {sol }}=4 V_{11} V_{11} V_{12} V_{12}
$$


Our ansatz can perfectly fit the experimental bounds as is shown by the following example.

We choose as input the masses for the charged leptons,

$$
m_{e}=0.511 \mathrm{MeV}, \quad m_{\mu}=105.7 \mathrm{MeV}, \quad m_{\tau}=1777 \mathrm{MeV}
$$

which correspond to the phases $a=0.0214$ and $b=0.2662$ of Eq.(13). For the neutrino sector, we choose,

$$
m_{\nu_{3}}=2 \mathrm{eV}, \quad \Delta m_{21}^{2}=9.2 \times 10^{-6} \mathrm{eV}^{2}, \quad \Delta m_{32}^{2}=5.0 \times 10^{-3} \mathrm{eV}^{2}
$$

This fixes the values of the parameters $\epsilon_{21}=3 \times 10^{-6}$ and $\epsilon_{32}=7.2 \times 10^{-4}$. With the above input we obtain for the leptonic mixing matrix $V$, without approximations:

$$
|V|=\left[\begin{array}{lll}
0.9976 & 0.0692 & 0.0058 \\
0.0463 & 0.6068 & 0.7935 \\
0.0518 & 0.7918 & 0.6085
\end{array}\right]
$$

Making use of Eqs.(29) and (31), Eq.(34) translates into

$$
\sin ^{2} 2 \theta_{\mathrm{atm}}=0.933
$$

and

$$
\sin ^{2} 2 \theta_{\text {sol }}=0.019
$$

These results are in agreement with the present experimental data. In this example the three physical neutrinos under consideration all have masses close to $2 \mathrm{eV}$. Note that the leptonic mixing matrix $V$, which differs significantly from the observed quark mixing matrix, was obtained having as input the charged lepton and neutrino masses, with no further parameters.

\section{Concluding remarks}

We have suggested an ansatz for the neutrino and charged lepton mass matrices, within the framework of universality of strength of Yukawa couplings. The ansatz has the same structure of phases for the neutrino and charged lepton mass matrices, with the only non-vanishing phases along the diagonal. Both $M_{\nu}$ and $M_{\ell}$ have only three parameters each, two phases and an overall real constant. These parameters are completely fixed by the value of charged lepton and neutrino masses, which implies that the ansatz is highly predictive, with full calculability of the leptonic mixing matrix, i.e., $V$ is completely fixed by charged lepton and neutrino mass ratios. We have shown that the ansatz naturally leads on the one hand to small mixing among $\nu_{e}$ and $\nu_{\mu}, \nu_{\tau}$ associated to $\Delta m_{21}^{2} \sim 10^{-5} \mathrm{eV}^{2}$ thus explaining the solar neutrino deficit in the non-adiabatic MSW solution, and on the other hand leads to large mixing between $\nu_{\mu}$ and $\nu_{\tau}$, as required to explain the atmospheric neutrino anomaly. 
We find our results specially appealing, since one has an unified view of all Yukawa couplings, i.e., both quark and lepton Yukawa couplings have universal strength, with the flavour dependence being all contained in their phases. Furthermore, both in the lepton and quark sectors [10] one has simple ansätze within USY, whose distinctive feature is having a number of independent parameters equal to the number of elementary fermion masses. As a result, one has highly predictive schemes, with the fermion mixings expressed in terms of fermion mass ratios with no free parameters.

\section{Acknowledgments}

We would like to thank M. Tanimoto for enlightening discussions during the preparation of this work.

\section{References}

[1] Y. Itow, Talk given at Topical Conference in SLAC Summer Institute, SLAC, USA, August 1997; K. S. Hirata et al., Nucl. Phys. B (Proc. Suppl.) 38 (1995) 55; B. T. Cleveland et al., Nucl. Phys. B (Proc. Suppl.) 38 (1995) 47; J. N. Abdurashitov et al., Phys. Lett. B328 (1994) 234: Phys. Rev. Lett. 77 (1996) 4708; P. Anselmann et al., Phys. Lett. B285 (1992) 376: B314 (1993) 445: B342 (1995) 440: B357 (1995) 237; W. Hampel et al., Phys. Lett. B388 (1996) 384.

[2] C. R. Proffitt, Ap. J. 425 (1994) 849; J. N. Bahcall and M. H. Pinsonneault, Rev. Mod. Phys. 64 (1992) 885: 67 (1995) 781; S. Turk-Chieze et al., Phys. Rep. 230 (1993) 57; A. Dar and G. Shaviv, Astrophys. J. 468 (1996) 933.

[3] L. Wolfenstein, Phys. Rev. D17 (1978) 2369: D20 (1979) 2634; S. P. Mikheev and A. Yu. Smirnov, Yad. Fiz. 42 (1985) 1441, [Sov. J. Nucl. Phys. 42 (1985) 913]: Nuovo Cim 9C (1986) 17; V. Barger et al., Phys. Rev. D22 (1980) 2718; H. A. Bethe, Phys. Rev. Lett. 56 (1986) 1305; S. P. Rosen and J. M. Gelb, Phys. Rev. D34 (1986) 969; J. Bouchez et al., Z. Phys. C32 (1986) 499.

[4] N. Hata and P. Langacker, Phys. Rev. D56 (1997) 6107.

[5] Y. Itow in Ref.[1]; K. S. Hirata et al., Phys. Lett. B205 (1988) 416: B280 (1992) 146; Y. Fukuda et al., Phys. Lett. B335 (1994) 237; R. Becker-Szendy et al., Phys. Rev. D46 (1992) 3720; D. Casper et al., Phys. Rev. Lett. 66 (1991) 2561; C. Berger et al., Phys. Lett. B227 (1989) 489: B245 (1990) 305; M. Aglietta et al., Europhys. Lett. 15 (1991) 559; W. W. M. Allison et al., Phys. Lett. B391 (1997) 491.

[6] C. Athanassopoulos et al., Phys. Rev. Lett. 75 (1995) 2650: 77 (1996) 3082: Phys. Rev. C54 (1996) 2685: nucl-ex/9709006. 
[7] J. R. Primack et al., Phys. Rev. Lett. 74 (1995) 2160.

[8] G. C. Branco, J. I. Silva-Marcos and M. N. Rebelo, Phys. Lett. B237 (1990) 446.

[9] G. C. Branco and J. I. Silva-Marcos, Phys. Lett. B359 (1995) 166.

[10] G. C. Branco, D. Emmanuel-Costa and J. I. Silva-Marcos, Phys. Rev. D56 (1997) 107.

[11] R. N. Mohapatra and S. Nussinov, Phys. Lett. B346 (1995) 75; P. F. Harrison, D. H. Perkins and W. G. Scott, Phys. Lett. B396 (1997) 186; C. D. Froggatt, M. Gibson and H. B. Nielson, Phys. Lett. B409 (1997) 305; P. Binétruy, S. Lavignac, S. Petcov and P. Ramond, Phys. Lett. B496 (1997) 3; T. Teshima, T. Sakai and O. Inagaki, hep-ph/9704266; M. Fukugita, M. Tanimoto and T. Yanagida, hep-ph/9709388; C. D. Froggatt, hep-ph/9712501.

[12] M. Gell-Mann, P. Ramond and R. Slansky in Sanibel Talk, CALT-68-709, Feb 1979 and in "Supergravity" edited by P. van Nieuwenhuizen and D. Freedmann, North-Holland, Amsterdam, 1979, p.315; T. Yanagida, Proceedings of the Workshop on "The Unified Theory and the Baryon Number of the Universe" edited by O. Sawada and A. Sugamoto, KEK 13-14 Feb 1979, Tsukuba (KEK-79-18).

[13] B. Pontecorvo, Zh. Eksp. Teor. Fiz. 33 (1957) 549: 34 (1958) 247; Z. Maki, N. Nakagawa and S. Sakata, Prog. Theor. Phys. 28 (1962) 870; V. Gribov and B. Pontecorvo, Phys. Lett. 28B (1969) 493; S. M. Bilenkii and B. M. Pontecorvo, Phys. Rep. 41 (1978) 225. 